\title{
Design Methodology for Controlled-Q Resonators in OTA-based Filters
}

\author{
Saeed Ghamari, Gabriele Tasselli, Cyril Botteron, and Pierre-André Farine \\ École Polytechnique Fédérale de Lausanne, ESPLAB, 2000 Neuchâtel, Switzerland
}

\begin{abstract}
This paper presents a design methodology for high quality factor resonators based on operational transconductance amplifier (OTA) employed in active filters. The quality factor of a resonator, as its main specification, is translated to the requirements of the OTA. Moreover, the effects of the OTA's finite output resistance and internal poles are investigated. The results provide a useful chart and a simple methodology to design a resonator with a desired quality factor. The design methodology has been validated by fabricating a resonator with $8 \mathrm{MHz}$ resonance frequency and a quality factor of around 10 using UMC 180-nm CMOS technology.
\end{abstract}

Index Terms - Gyrators, resonator filters, operational transconductance amplifier (OTA), quality factor (Q), active inductors, active filters.

\section{INTRODUCTION}

System-on-chip wireless communication systems, such as LTE cellular, GNSS and UWB require more and more bandwidth (BW) and selectivity due to the growing number of users and systems working together [1]-[4]. These requirements thus also apply to the constituent continuoustime filters. These filters can be realized by means of passive components such as resistors, capacitors and inductors. However, passive inductors in complementary metal-oxide-semiconductor (CMOS) technology are bulky and suffer from a low quality factor (Q). A well-known solution to this problem consists of adopting an active inductor which occupies less chip area and provides a variable large inductance as well as a tunable Q. Active inductors can be realized by loading a gyrator with a capacitor and are commonly used in active filter design [5].

Gyrators usually consist of two identical operational transconductance amplifiers (OTAs) which are connected together in a negative feedback loop. However, OTAs nonidealities such as limited output impedance and internal poles degrade the $\mathrm{Q}$ as well as the frequency response of both the inductors and filters. Limited output impedance results in a finite DC gain for an OTA, which then limits the maximum $\mathrm{Q}$ of the resonator [6]. Moreover, the internal poles of the OTA can produce a phase shift of the signal in the loop of the gyrator. At high frequencies, this phase shift reduces the phase margin (PM) around the biggest internal pole of the OTA which is called the second pole of the OTA and can cause unwanted peaking in the gain and thus alter the $\mathrm{Q}$ of the resonator.

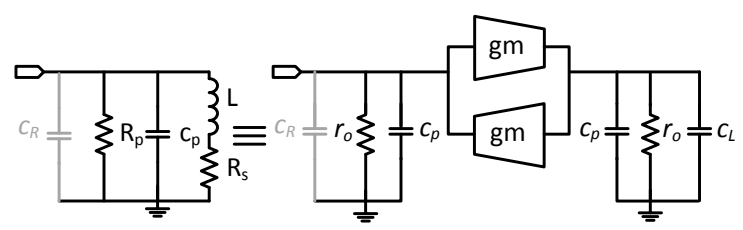

Fig. 1. A resonator consists of an active inductor (in black) and a loading capacitance $c_{R}$ with its equivalent RLC circuit; $c_{p}$ and $r_{o}$ are output parasitic capacitance and output resistance of the OTA, respectively.

This paper elaborates on the effects of OTA's nonidealities on the resonator's $\mathrm{Q}$ by means of simulations. Techniques to meet the resonator's specification are described and a simple methodology is presented. The proposed methodology has been validated by simulations and measurements. For this purpose, a resonator with $8 \mathrm{MHz}$ resonance frequency and $\mathrm{Q}$ of 10 has been designed and fabricated using UMC 180-nm CMOS technology.

\section{From Resonator Specifications to OTA REQUIREMENTS}

OTA-based resonators are commonly used in active filter design, thus their specifications are imposed by the filter requirements. In other words, the specifications of the filter translate to the specifications of the OTA inside the resonator. Since the performance of the filter depends on the most selective resonator of the filter, we will focus in this section on the specifications of a resonator such as $\mathrm{Q}$ and resonance frequency $\omega_{0}$ and translate them to the specifications of an OTA. An active inductor which is constructed from two identical OTAs and a capacitor $c_{L}$ is shown in Fig. 1 along with its equivalent circuit. Assuming that, in a narrow band system, the OTA has a constant transconductance of $g_{m}$, finite output resistance of $r_{o}=1 / g_{o}$ and an input and output parasitic capacitance $c_{p}$, the equivalent circuit's parameters are

$$
L=\frac{c_{L}+c_{p}}{g_{m}^{2}}, \quad R_{s}=\frac{g_{o}}{g_{m}^{2}}, \quad R_{p}=\frac{1}{g_{o}} .
$$

To examine the $\mathrm{Q}$ and $\omega_{0}$ of a resonator, consider the case in which a capacitor $c_{R}$ is connected in parallel to the active inductor of Fig. 1 to obtain the resonator. The RLC network impedance can be written as: 


$$
Z(s)=\frac{\frac{s L+R_{s}}{\left(c_{R}+c_{p}\right) L}}{s^{2}+s\left(\frac{1}{R_{p}\left(c_{R}+c_{p}\right)}+\frac{R_{s}}{L}\right)+\frac{R_{s}+R_{p}}{R_{p}\left(c_{R}+c_{p}\right) L}} .
$$

Considering complex conjugate poles for the resonator, its resonance frequency can be written as:

$$
\omega_{0}=\sqrt{\frac{R_{s}+R_{p}}{R_{p}\left(c_{R}+c_{p}\right) L}}=\sqrt{\frac{g_{o}^{2}+g_{m}^{2}}{\left(c_{R}+c_{p}\right)\left(c_{L}+c_{p}\right)}} .
$$

It is clear that the input parasitic capacitance and the finite output resistance of the OTA cause a frequency shift in the resonance frequency of the resonator and increase the active inductor value. Assuming $c_{p}=0$ ( $c_{p}$ can still be modeled with $c_{R}$ and $\left.c_{L}\right), c_{R}=c_{L}$ and $g_{m} \gg g_{o}$, the resonance frequency in (3) will be equal to the gain-bandwidth product (GBW) of the OTA. The main specification of a resonator is its $\mathrm{Q}$; the other specifications such as noise in active inductor and the resonator's dynamic range pertain to that [6]. Based on the definition of Q in [7], from (1) and (2) and considering complex conjugate poles for the resonator, the $\mathrm{Q}$ of the resonator can be written as

$$
Q \triangleq \frac{\omega_{0}}{\frac{1}{R_{p}\left(c_{R}+c_{p}\right)}+\frac{R_{s}}{L}}=\frac{\sqrt{\left(g_{o}^{2}+g_{m}^{2}\right)\left(c_{R}+c_{p}\right)\left(c_{L}+c_{p}\right)}}{g_{o}\left(c_{R}+c_{L}+2 c_{p}\right)}
$$

The maximum value of (4) occurs when $c_{L}=c_{R}$ $\left(Q=\sqrt{\left(g_{o}^{2}+g_{m}^{2}\right)} / 2 g_{o}\right)$, and in this case with a realistic assumption of $g_{m} \gg g_{o}$, the maximum Q will be half of the DC gain of the OTA $\left(Q=g_{m} / 2 g_{o}\right)$. Therefore, to design a resonator with quality factor of $\mathrm{Q}$ with an OTAbased gyrator, the gain of the OTA should be at least twice the required $\mathrm{Q}$.

Moreover, internal poles of the OTA cause a phase shift in the signal through the OTA and in the loop of the gyrator this phase shift can become a problem at high frequencies. In that case the active inductor shows a negative resistance and causes peaking in the $\mathrm{Q}$ of the resonator as well as in its frequency response. The second pole of the OTA has the main contribution in this effect. To elaborate more in this effect, two simulations have been carried out by employing an OTA with one internal pole at $\omega_{1}$. First, we simulated a resonator to see the variation of its $Q$ over different positions of $\omega_{1}$ then we simulated the active inductor of the resonator to see when it shows a negative resistance. The active inductor in these simulations is the same as the one in Fig. 1 but with the assumption of no parasitic capacitance $\left(c_{p}=0\right)$. The resonator uses the same inductor loaded by the capacitance $c_{\mathrm{L}}\left(c_{R}=c_{L}\right.$ in Fig. 1). The simulation results are shown in Fig. 2 and Fig. 3.

Fig. 2 shows the variation of $Q \triangleq \omega_{0} / \Delta \omega$ of the resonator versus the position of $\omega_{1}$ regardless of values of $Q_{i}$, where $\omega_{0}$ is the resonance frequency of the resonator,

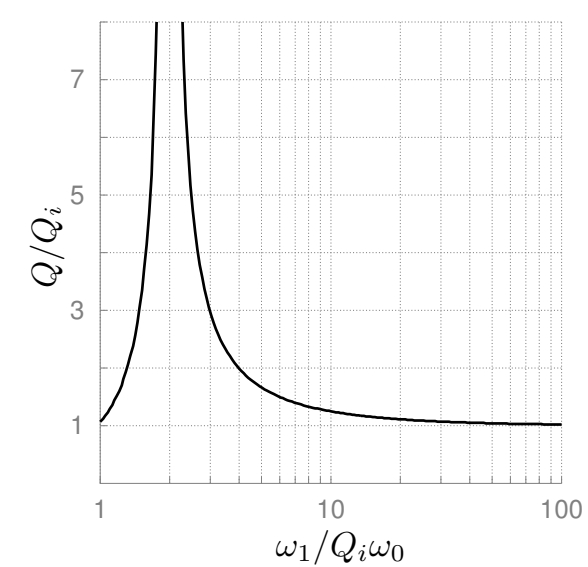

Fig. 2. Design chart of the variation of the resonator's $Q$ versus sweeping the second pole $\left(\omega_{1}\right)$ of the OTA; $Q_{i}$ is the Q when the OTA does not have the second pole (i.e. $\omega_{1} \rightarrow \infty$ ) and $\omega_{0}$ is the resonance frequency of the resonator.

$\Delta \omega$ is the 3-dB BW of the resonator and $Q_{i}$ is the $\mathrm{Q}$ when the OTA does not have any internal pole $\left(\omega_{1} \rightarrow \infty\right)$. Fig. 2 indicates that, regardless of the value of $Q_{i}$ and $\omega_{0}$, around $\omega_{1}=2 Q_{i} \omega_{0}$, the $\mathrm{Q}$ shows a huge peaking in its value. The figure can be used as a chart to locate the $\omega_{1}$ in the OTA design process based on the amount of tolerable variation of the Q of a resonator. For example, for $\omega_{1}=4 Q_{i} \omega_{0}$, the $\mathrm{Q}$ is twice the value that was intended $\left(Q_{i}\right)$. This figure also shows that to keep the variation of $\mathrm{Q}$ within $25 \%, \omega_{1}$ should be placed at least at $10 Q_{i} \omega_{0}$.

The variation of $\mathrm{Q}$ in Fig. 2 is due to the negative resistance that the inductor shows because of its internal pole. Fig. 3 indicates when the active inductor starts to show negative resistance for a different location of $\omega_{1}$. The $\omega_{N}$ in this figure is the zero-resistance frequency of the active inductor which for frequencies lower and higher than $\omega_{N}$ the inductor shows positive and negative resistance, respectively. To be able to compare Fig. 3 with Fig. 2, $\omega_{N}$ and $\omega_{1}$ are normalized by $\omega_{0}$ and $Q_{i}$ of the resonator of Fig. 2. Looking at $\omega_{1}=2 Q_{i} \omega_{0}$ in Fig. 3, one can see that the zero resistance happens at $\omega_{N}=\omega_{0}$. This means that the inductor does not show any resistance and that is why the resonator shows a huge peaking of $\mathrm{Q}$ for the same location of the internal pole.

Another simulation was carried out to show the effect of the variation of $\mathrm{Q}$ over the position of the second pole of the OTA on the resonator response for three different resonance frequencies $(18.7,21,23 \mathrm{MHz})$. The results are shown in Fig. 4. The resonator was designed to have the $Q_{i}=20$ and the second pole of the OTA was located at 3 and 80 times the $Q_{i} \omega_{0}$ of Fig. 2. Furthermore, the resonator's response for one resonance frequency in ideal case when the OTA has no internal pole $\left(\omega_{1} \rightarrow \infty\right)$ is also shown in Fig. 4. As was expected from Fig. 2, the $Q$ of the 


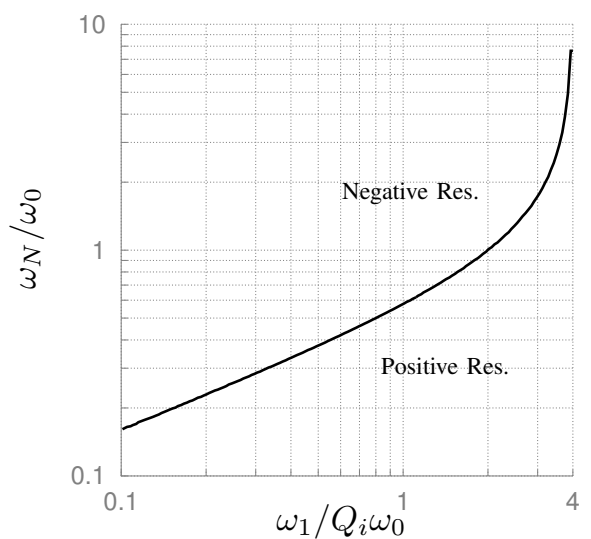

Fig. 3. The zero-resistance frequency $\left(\omega_{N}\right)$ of the active inductor versus sweeping the second pole $\left(\omega_{1}\right)$ of the OTA; $\omega_{0}$ is the resonance frequency of the resonator that is made by the active inductor loaded by capacitance $\mathrm{c}_{\mathrm{L}}$, and $Q_{i}$ is the $\mathrm{Q}$ of the resonator when $\omega_{1} \rightarrow \infty$.

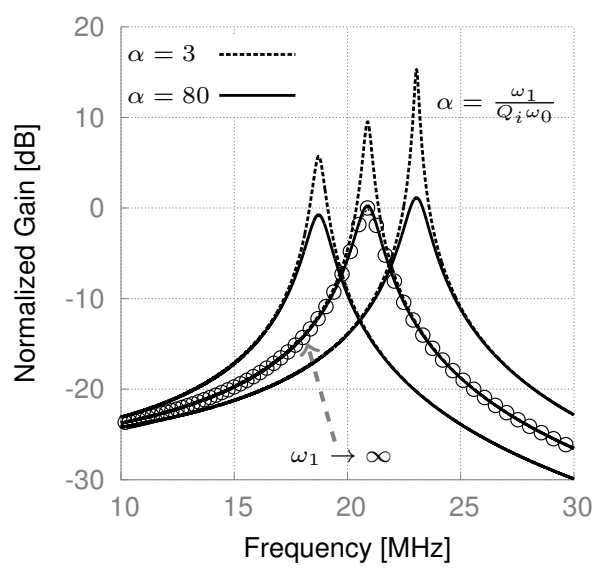

Fig. 4. Variation in gain and $\mathrm{Q}$ with tuning of $\omega_{0}$ (at 18.7, 21, $23 \mathrm{MHz}$ ) for the two cases of $\omega_{1}$ at $3 Q_{i} \omega_{0}$ and $80 Q_{i} \omega_{0}$; along with the resonator response in ideal case when the OTA has no internal pole $\left(\omega_{1} \rightarrow \infty\right)$.

resonator made by the OTA with its second pole at $80 Q_{i} \omega_{0}$ is coincident with the ideal case. However, there is some peaking in the resonator response for the other case, which causes higher values of Q. Even though a higher value of $\mathrm{Q}$ is usually wanted, this $\mathrm{Q}$ is not constant over the tuning of the resonator (see Fig. 4). Therefore, Fig. 2 can be utilized to locate the second pole of the OTA in the design process of the resonator based on the tolerable variation of the $\mathrm{Q}$. However, since process, voltage and temperature (PVT) variation effects can cause slightly shift in $\omega_{1}$, thus high ratio of $Q / Q_{i}$ where the $\mathrm{Q}$ of a resonator is very sensitive to $\omega_{1}$, should be avoided for a design.

\section{Circuit Implementation}

This section presents the design methodology for a resonator with $\mathrm{Q}$ of 10 and resonance frequency at $8 \mathrm{MHz}$ which was fabricated using UMC 180-nm CMOS technology. The photograph of the test chip is shown in Fig. 5.

The design procedure starts with choosing a value for

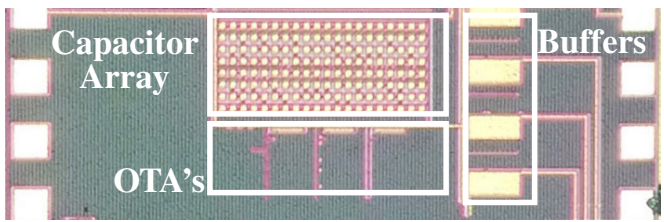

Fig. 5. Photograph of the resonator implemented in UMC 180nm CMOS technology; the resonator occupies $370 \times 475 \mu \mathrm{m}$.

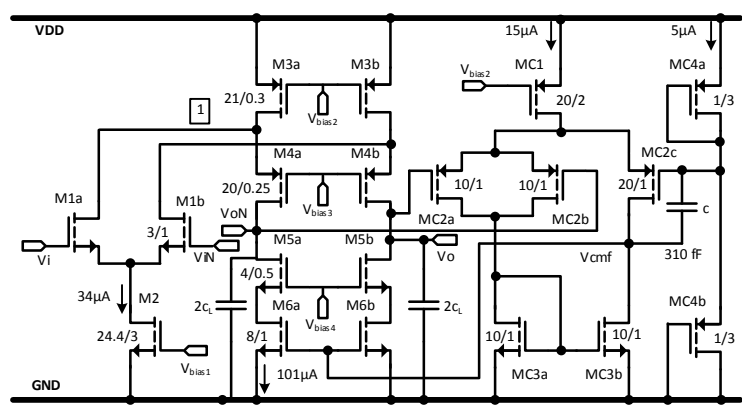

Fig. 6. Schematic of the folded cascode OTA.

the loading capacitance since all the parameters of the resonator are related to that. Usually the minimum value of the capacitor is set by the noise requirement of the resonator [8] and based on this fact, a differential load of $\mathrm{c}_{\mathrm{L}}=1.18 \mathrm{pF}$ was chosen in this design. The next step is to design a gyrator which consists of two identical OTAs.

The OTA's topologies are well explained in [9] and the folded cascode topology was preferred for this design due to advantages that it offers over the others. One of these advantages which is very important in resonator design, is that the second pole is located at very high frequency compared to other topologies, i.e. telescopic or symmetrical OTA, without any extra compensation techniques. The schematic of the folded cascode is shown in Fig. 6.

The next step is to set the value of $\omega_{0}=2 \pi f_{0}$ and Q. $\omega_{0}$ is set by the transconductance of differential pair M1 $\left(g_{m 1}\right)$. For the first-guess design, from (3) and assuming that $g_{m 1} \gg g_{o 1}$ and $c_{L}=c_{R} \gg c_{p}$, for $f_{0}=8 \mathrm{MHz}$ and $\mathrm{c}_{\mathrm{L}}=1.18 \mathrm{pF}, g_{m 1}$ can be calculated as $60 \mu \mathrm{S}$. To size M1, its overdrive voltage also needs to be set. This voltage affects the linearity of the resonator, for low power design, overdrive voltage of a differential pair can be chosen around $200-300 \mathrm{mV}$ [8]. In this case, $300 \mathrm{mV}$ is chosen as the differential pair overdrive. The overdrive voltage along with the $g_{m 1}$ set the bias current of M1a and M1b around $18 \mu \mathrm{A}$. To have a good trade off between the gain and the transition frequency $\left(f_{T}\right)$ of the differential pair, the length of M1 was chosen as $1 \mu \mathrm{m}$ and its width was chosen to provide the bias current.

After the design of the differential pair, the next step is to design the output stage of the OTA which is associated to the $\mathrm{Q}$ of the resonator. As it was discussed in section II, in the OTA-based resonator the DC gain of the OTA limits the maximum $\mathrm{Q}$ of the resonator and the second pole of 
the OTA must be placed far beyond its GBW. These are the two main criteria which should be considered to design the output stage of the OTA. In this case, to save power a maximum variation of $25 \%$ of $\mathrm{Q}$ is acceptable and the $Q_{i}$ is chosen as 10. Therefore, from Fig. 2 the second pole of the OTA should be placed at $f_{1}=10 Q_{i} f_{0}=800 \mathrm{MHz}$ to result a $\mathrm{Q}$ of 10 with $25 \%$ tolerance for the resonator. The second pole frequency of the OTA in Fig. 6 can be written as $f_{1}=g_{m 4} /\left(4 \pi C_{n 1}\right)$, where $g_{m 4}$ is the transconductance of the transistor M4 and $C_{n 1}$ is the parasitic capacitance at node 1 which can be written as $C_{n 1}=C_{G S 4}+C_{D B 1}+C_{D B 3}$. Therefore, to have $f_{1}$ at around $800 \mathrm{MHz}$, a high value of $g_{m 4}$ is needed as well as low parasitic capacitance at node 1 . The latter has been achieved by choosing the short channel of $300 \mathrm{~nm}$ and $250 \mathrm{~nm}$ for M3 and M4, respectively. The bias current can be set by considering the required slew rate. In this design since the resonator might be needed to be tuned till $20 \mathrm{MHz}$, to satisfy the slew rate for $400 \mathrm{mV}$ of output swing at maximum frequency of $20 \mathrm{MHz}$, a bias current greater than $100 \mu A$ is needed. In this case, the low overdrive for M4 helps to increase the $g_{m 4}$; the $g_{m 4}=1 \mathrm{mS}$ has been achieved by an overdrive of $230 \mathrm{mV}$. Transistor M3 is $21 \mu \mathrm{m}$ wide to provide the current for the input and output stage of the OTA. Moreover, its overdrive is adjusted to tune the $g_{m 1}$ hence $f_{0}$. The simulated parasitic capacitance of $C_{n 1}$ is about $90 \mathrm{fF}$ which places the second pole of the OTA at $880 \mathrm{MHz}$. In the active load, M5 has a short channel to have high $f_{T}$ while M6 is designed for common-mode (CM) control. Also, the load capacitor of $c_{\mathrm{L}}$ is implemented with a $2 \mathrm{c}_{\mathrm{L}}$ capacitor connected to the ground to provide the path also for the CM signal. The transistors' size and bias currents are optimized in Cadence $^{\circledR}$ Spectre ${ }^{\circledR}$ simulator and the final values are shown in Fig. 6.

The resonator was fabricated and it consumes $520 \mu \mathrm{A}$ from $1.8 \mathrm{~V}$ resonating at $8 \mathrm{MHz}$. The measured responses of the resonator for the resonance frequencies at 8,15 and

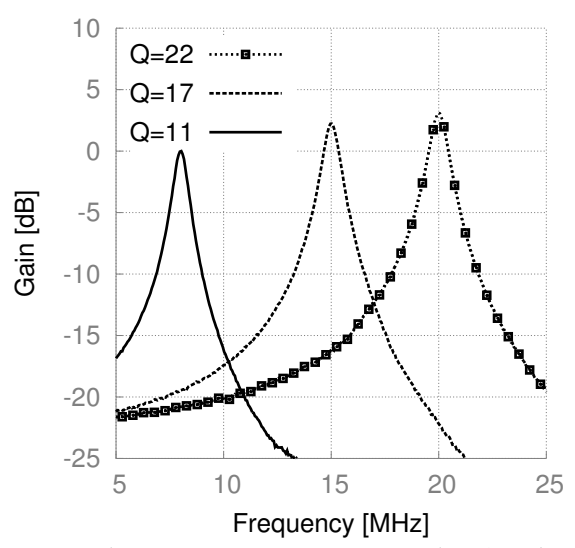

Fig. 7. Measured resonator responses tuned at 8, 15 and $20 \mathrm{MHz}$.
$20 \mathrm{MHz}$ are shown in Fig. 7. Considering $f_{1}=880 \mathrm{MHz}$ and $Q_{i}=10$, the resonator shows an expected $\mathrm{Q}$ of 11 at $8 \mathrm{MHz}$ which fully corresponds to $\omega_{1} / Q_{i} \omega_{0}=11$ in Fig. 2. Furthermore, the $\mathrm{Q}$ at other resonance frequencies were also predictable from Fig. 2 because for resonance frequencies at 15 and $20 \mathrm{MHz}, \omega_{1} / Q_{i} \omega_{0}$ are equal to 5.8 and 4.4, which correspond to a $\mathrm{Q}$ of 16 and 19 from Fig. 2, respectively. Considering the PVT variations, the measured results are therefore well in agreement with Fig. 2, which validates the use of Fig. 2 for the proposed design methodology.

\section{CONCLUSION}

The design methodology of an OTA-based resonator was presented in this paper. The resonator's Q and resonance frequency were translated to the specifications of the OTA. Furthermore, a comprehensive analysis on the effect of the OTA's internal pole on the resonator's Q was carried out by means of simulations. It showed that the second pole of the OTA has a direct influence on the resonator's $\mathrm{Q}$ and its variation in response to tuning the resonator's resonance frequency $\left(\omega_{0}\right)$. As a rule of thumb, placing the second pole beyond $10 Q \omega_{0}$ keeps the variation of the $\mathrm{Q}$ within $25 \%$. To validate the proposed approach, a resonator using the proposed methodology was fabricated. The measurement results show a $\mathrm{Q}$ of 11 slightly above the required $\mathrm{Q}$ of 10 resonating at the correct resonance frequency of $8 \mathrm{MHz}$ which was expected from Fig. 2.

\section{REFERENCES}

[1] N. Qi et al., "A Dual-Channel Compass/GPS/GLONASS/Galileo Reconfigurable GNSS Receiver in $65 \mathrm{~nm}$ CMOS With On-Chip I/Q Calibration," IEEE Trans. Circuits Syst. I Regul. Pap., vol. 59, no. 8, pp. 1720-1732, Aug. 2012.

[2] H. Xie et al., "Single-Chip Multiband EGPRS and SAWLess LTE WCDMA CMOS Receiver With Diversity," IEEE Trans. Microw. Theory Tech., vol. 60, no. 5, pp. 1390-1396, May 2012.

[3] D. Morche et al., "Double-Quadrature UWB Receiver for Wide-Range Localization Applications With Sub-cm Ranging Precision," IEEE J. Solid-State Circuits, vol. 48, no. 10, pp. 2351-2362, Oct. 2013.

[4] F. Chastellain, C. Botteron, and P.-A. Farine, "Looking Inside Modern Receivers," IEEE Microw. Mag., vol. 12, no. 2, pp. 87-98, Apr. 2011.

[5] F. Yuan, "CMOS gyrator-C active transformers," IET Circuits, Devices Syst., vol. 1, no. 6, p. 494, 2007.

[6] Y.-T. Wang and A. Abidi, "CMOS active filter design at very high frequencies," IEEE J. Solid-State Circuits, vol. 25, no. 6, pp. 1562-1574, 1990.

[7] W. M. Siebert, Circuits, Signals, and Systems. MIT Press, 1985.

[8] H. Khorramabadi and P. Gray, "High-frequency CMOS continuous-time filters," IEEE J. Solid-State Circuits, vol. 19, no. 6, pp. 939-948, Dec. 1984.

[9] W. M. C. Sansen, Analog Design Essentials, ser. The International Series in Engineering and Computer Science. Boston, MA: Springer US, 2006, vol. 859. 